\title{
High Resolution Pedogenic and Rock Magnetic Records of Asian Summer Monsoon Instability during the past 60,000 Years from the Chinese Western Loess Plateav
}

The $23 \mathrm{~m}$ Shajinping loess section on the second terrace of Huang $\mathrm{He}$ (the Yellow River) in the City of Lanzhou has developed at least 20 layers of moderately to weakly developed paleosols each 30-50 cm in thickness, occurring at similar intervals of about $1 \mathrm{~m}$ (ca. 2,500 years), with several very weakly developed soil horizons, during the past 60,000 years BP Fig 1). Field observations and laboratory analyses, including soil micromorphology, have indicated that Holocene paleosols are weakly to moderately developed luvic calcisols in type, characterized by a distinct dull brown (7.5 YR 5/3) medium angular blocky ped Bw horizon relatively rich in organic matter and clay content and low in carbonates (round $9 \mathrm{wt} \%$ ), and a clear massive Bk horizon, with carbonates up to $16 \mathrm{wt} \%$ as fine nodules, channel coatings and infillings. Paleosols formed

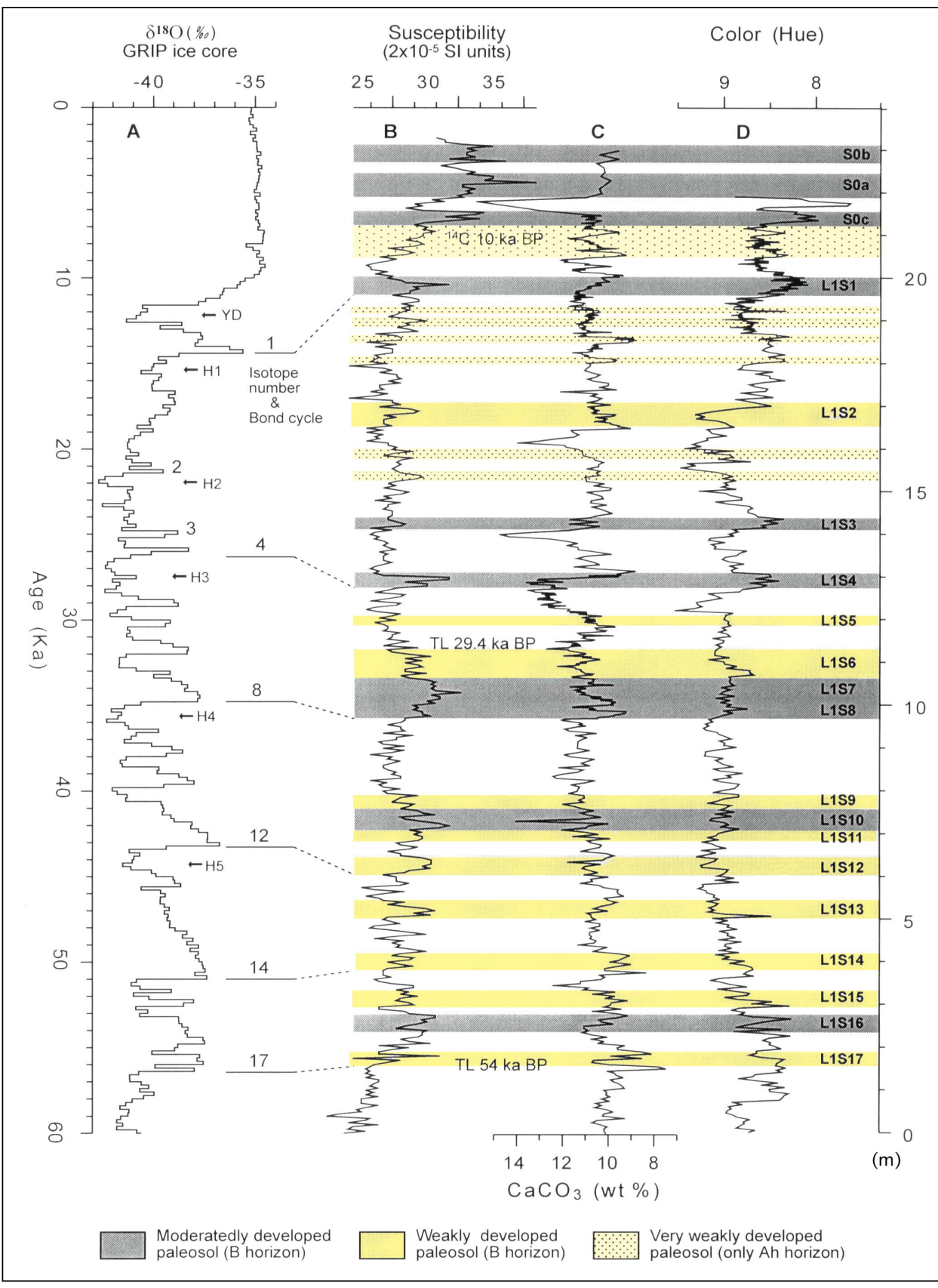

Fig. 10: Correlation of soil formation and summer monsoon enhancements revealed by magnetic susceptibility (B), carbonate content (C) and soil color (D) in the Shajinping loess section, Lanzhou, with the climatic record from the GRIP ice core in Greenland (A). Paleosol identification was independently made in field. H indicates Heinrich event. Several meters of the lower part of the section may have been affected somewhat by overbank flooding. during Marine Isotope Stages 2, 3 and 4 are mainly weakly developed and appear as thin, dull, yellowish brown (10 YR 5/4 - 4/3) massive-medium blocky ped ochric A horizons, or mollic A horizons slightly rich in organic matter and clay content and low in carbonates (round 9-10 wt \%) in the form of fine nodules and thin coatings. However, there are six moderately developed paleosols pedogenically similar to the Holocene paleosols. The organic matter and clay content in the Bw horizons and carbonate enrichment in the Bk horizons are only slightly lower than in the Holocene paleosols. These six paleosols occur at similar intervals of 3-4 m (about half of the precession cycle, i.e. 10,000 + / - 1000 years) and seem to correlate well with the initial warm peaks of each Bond cycle (Fig. 10).

Continuous sampling of the section at $2.5-5 \mathrm{~cm}$ intervals for measurement of carbonate content and magnetic susceptibility has yielded decadal- to century-scale climatic records. These records demonstrate that the Asian summer monsoon experienced rapid episodic 'warm pulses' lasting only about several hundred to two thousand years during Isotope Stages 2 and 3 . These warm pulses form groups characterized by high initial, then subsequently declining peaks. They may correlate with 'warm' peaks in the oxygen isotope record from the GRIP ice core. Most of these warm pulses occur in non- or quasi-orbital forcing cycles and have pedogenic responses as described above. This provides evidence for changes in the Asian summer monsoon on millennial timescales during the last glacial and points to instability similar to that identified by the Dansgaard-Oeschger events and Bond cycles in the Northern Atlantic.

Xiao-Min Fang, Bao-TianPan, Ji-Jun Li, Dong-Hong Guan

Department of Geography, Lanzhou University, Lanzhou, Gansu 730000, China

Yugo Ono

Graduate School of Environmental Earth Science, Hokkaido University, Sapporo, 060 Japan

Hitoshi FukusaWa

Department of Applied Biochemistry, University of Tsukuba, Tennou-dai 1-1-1, Tsukuba, 305 Japan

Shizuo Nagatsuka

Department of Geography, Faculty of Science, Tokyo Metropolitan University, Hachioji, 192-03 Japan

MasaYuKI TORI

Division of Earth and Planetary Sciences, Graduate School of Sciences, Kyoto University,

Kyoto, 606-01 Japan 